\title{
Mechanoreceptor-mediated circuit regulates cold tolerance in Caenorhabditis
} elegans

Natsune Takagaki ${ }^{1-2}$, Akane Ohta ${ }^{1-3} *$, Kohei Ohnishi ${ }^{1-2}$, Yohei Minakuchi ${ }^{4-5}$, Atsushi Toyoda $^{4-5}$, Yuichiro Fujiwara ${ }^{6} \&$ Atsushi Kuhara ${ }^{1-3,7 *}$

${ }^{1}$ Graduate School of Natural Science, Konan University, Kobe 658-8501, JAPAN

${ }^{2}$ Institute for Integrative Neurobiology, Konan University, Kobe 658-8501, JAPAN

${ }^{3}$ Faculty of Science and Engineering, Konan University, Kobe 658-8501, JAPAN

${ }^{4}$ Advanced Genomics Center, National Institute of Genetics, Mishima, Shizuoka 411-8540, JAPAN

${ }^{5}$ Comparative Genomics Laboratory, National Institute of Genetics, Mishima, Shizuoka 411-8540, JAPAN

${ }^{6}$ Laboratory of Molecular Physiology \& Biophysics, Faculty of Medicine, Kagawa University, Kagawa 761-0793, JAPAN

${ }^{7}$ AMED-PRIME, Japan Agency for Medical Research and Development, Tokyo 100-0004, JAPAN

*Please address correspondences and requests to A.K. \& A.O.

Tel/Fax: +81-78-431-4341

E-mail: atsushi_kuhara@me.com (A.K.), aohta@center.konan-u.ac.jp (A.O.)

Running title: Mechanoreceptor-mediated cold tolerance

Teaser: Thermosensitive mechanoreceptors regulate cold tolerance in Caenorhabditis elegans. 
Takagaki et al.

\begin{abstract}
C. elegans mechanoreceptors located in the ASG sensoryneuron have been found to sense temperature - a key trait for animal survival. Experimental loss of xanthine dehydrogenase (XDH-1) function in the AIN and AVJ interneurons resulted in reduced cold tolerance and atypical neuronal response to changes in temperature. These interneurons are synapse with upstream neurons such as the mechanoreceptor-expressing ASG. $\mathrm{Ca}^{2+}$ imaging revealed that ASG responsiveness to temperature change via mechanoreceptor DEG-1, a Degenerin/Epithelial Sodium Channel (DEG/ENaC), affects downstream AIN and AVJ circuits. Ectopic expression of DEG-1 in the ASE gustatory neuron resulted in acquisition of thermosensitivity, while electrophysiological analysis revealed that DEG-1 was involved in temperature sensation. Together, these results suggest that cold tolerance is regulated by mechanoreceptor-mediated circuit calculation.
\end{abstract}

Key words: C. elegans / cold tolerance / mechanoreceptor / temperature sensation; 
Takagaki et al.

\section{Introduction}

Animal temperature detection has previously been studied with a focus on transient receptor potential (TRP) channels. TRPV1, for example, is known to detect regions of high temperature, while TRPA1 detects regions of low temperature (Dhaka, Viswanath et al., 2006). Regarding TRP-independent temperature detection pathways, G proteincoupled receptor (GPCR)/rhodopsin in drosophila may act as a temperature receptor able to modulate decision-making behavior (Shen, Kwon et al., 2011). Furthermore, receptor-type guanylyl cyclases (rGCs) in the nematode worm C. elegans are thought to function as temperature receptors in the AFD temperature-sensing neuron given that ectopic expression of rGCs can confer temperature-dependent responses to heterologous cells (Takeishi, Yu et al., 2016).

C. elegans is an ideal model for the study of neural circuitry underlying cold tolerance given its simple nervous system composed of only 302 neurons as well as the number of well-studied molecular and genetic approaches currently available (Brenner, 1974). Mutant experimentation has also been well-documented, allowing for the identification of key genes and determination of specific neuron action sites (Barr, 2003). Finally, C. elegans temperature response has been analyzed with respect to many phenomena, including dauer larva formation (Barr, 2003), thermotactic behavior (Ohta \& Kuhara, 2013), and cold tolerance(Ohta, Ujisawa et al., 2014, Okahata, Ohta et al., 2016, Sonoda, Ohta et al., 2016, Ujisawa, Ohta et al., 2018).

Together, the literature suggests that $C$. elegans possesses an adaptive mechanism to tolerate cold external environments. Wild-type worms grown at $15^{\circ} \mathrm{C}$ can 
Takagaki et al.

survive at a temperature of $2^{\circ} \mathrm{C}$, whereas $20^{\circ} \mathrm{C}$ cultivated worms cannot (Figure 1A) (Ohta et al., 2014, Sonoda et al., 2016, Ujisawa et al., 2018). Nematode cold tolerance is a process that involves a number of tissues, including the ASJ and ADL sensory neurons, intestinal cells, sperm, and muscle cells (Ohta et al., 2014, Sonoda et al., 2016, Ujisawa et al., 2018). In terms of sequence and site, the process begins when temperature is detected by the ASJ and ADL neurons located in the head (Ohta et al., 2014, Ujisawa et al., 2018). Next, insulin is released from the ASJ and binds to insulin receptors in the intestine and nervous tissue (Ujisawa et al., 2018), which initiates steroid-hormonal signaling to the sperm. Sperm in turn modulate ASJ neuronal activity in a feedback-like manner (Ohta et al., 2014). Genes are later expressed that ultimately modify bodily lipid composition (Murray, Hayward et al., 2007), which is considered central to cold tolerance. However, this mechanism describes negative regulation while overlooking yet-unexplored positive regulation.

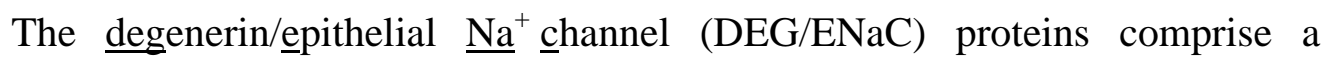
diverse family of sodium ion channels (Chen, Bharill et al., 2016, Geffeney, Cueva et al., 2011, Waldmann, Champigny et al., 1996) involved in cellular functions such as mechanosensation (Geffeney et al., 2011, Zhong, Hwang et al., 2010), sour/salt taste (Chandrashekar, Kuhn et al., 2010, Liu, Leonard et al., 2003, Ugawa, Yamamoto et al., 2003), learning, memory, and synaptic plasticity (Wemmie, Askwith et al., 2003, Wemmie, Chen et al., 2002, Wemmie, Coryell et al., 2004, Zha, Wemmie et al., 2006, Ziemann, Allen et al., 2009). In mammals, the DEG/ENaC channel MDEG is abundantly expressed in the brain. (Waldmann et al., 1996), while the C. elegans 
Takagaki et al.

homolog DEG-1 is expressed in association with multiple sensory neuron mechanoreceptors (Geffeney et al., 2011, Hall, Gu et al., 1997, Wang, Apicella et al., 2008). Although a decrease in temperature results in a change to the $\mathrm{Na}^{+}$potential across MDEG (Askwith, Benson et al., 2001), it remains unknown whether $\mathrm{DEG} / \mathrm{ENaCs}$ are directly involved in the temperature sensation process.

In mammals, xanthine oxidoreductase (XOR) is present as two interconvertible forms: xanthine dehydrogenase (XDH) and xanthine oxidase (XO). Moreover, both enzymes are active in the purine base salvage pathway. Notably, XDH converts hypoxanthine to xanthine while also oxidizing xanthine to urate. NADH is also produced simultaneous during the final step of purine salvage (Xi, Schneider et al., 2000).

$\mathrm{XDH}$ is expressed in the liver and small intestine (Chung, Baek et al., 1997). In humans, $\mathrm{XDH}$ expression levels are associated with tumor growth. Elevated expression of $\mathrm{XDH}$ is associated with tumor infiltration as well as upregulated proinflammatory and immune cytokine expression (Saidak, Louandre et al., 2018). $\mathrm{XDH}$ served as a useful biological parameter in one pan-cancer study (Saidak et al., 2018), yet the molecular function of XDH remains largely unexplored given that it may be either reversibly or irreversibly converted to XO in mammals (Saksela, Lapatto et al., 1999, Terawaki, Murase et al., 2017). Since invertebrate XOR is present only as XDH (Terawaki et al., 2017), C. elegans is thought to be a useful model.

We found that $x d h-1$ mutant xanthine dehydrogenase knock-out worms 
Takagaki et al.

exhibit abnormal cold tolerance, and that normal function can be recovered by expressing $x d h-1 c D N A$ in both $\mathrm{AIN}$ and $\mathrm{AVJ}$ interneurons. In vivo $\mathrm{Ca}^{2+}$ imaging revealed that $\mathrm{XDH}-1$ acts as a positive temperature signal regulator in $\mathrm{AIN}$ and as a negative regulator in AVJ, and that temperature sensation by ASG sensoryneuron via mechanoreceptor DEG-1 affects neural activity of both AIN and AVJ interneurons. Ectopic expression of DEG-1 in the ASE, a non-thermosensitive chemosensory neuron, resulted in acquisition of temperature sensation. In addition, two-electrode voltage-clamp recording of Xenopus oocytes expressing DEG-1 demonstrated thermoreceptor-like behavior. These results together suggest that DEG-1, a DEG/ENaC type mechanoreceptor, acts as a temperature receptor and is required for the neural circuit calculation of the positive regulation of cold tolerance.

\section{Results and Discussion}

To identify novel genes involved in cold tolerance, we isolated and analyzed the $c h r l$ mutation as characterized by decreased cold tolerance following cultivation at $15^{\circ} \mathrm{C}$ (Figures 1B and S1A-H, see supplemental material). Deep DNA sequencer and SNP analysis allowed the chrl mutation to be mapped from $6.13 \mathrm{cM}$ to $16.24 \mathrm{cM}$ on chromosome IV, where four genes were found to exhibit major mutations (Figures S1D-F) (accession number DRA: DRA 002599). We then evaluated cold tolerance in mutants for these genes (Figure 1B), with only $x d h-1$ mutants exhibiting markedly abnormal cold tolerance after cultivation at $15^{\circ} \mathrm{C}$. Moreover, abnormal cold tolerance in 
Takagaki et al.

both $c h r l$ and $x d h-1$ mutants was rescued by the expression of wild-type $x d h-1$ (Figures $1 \mathrm{C}$ and $\mathrm{D})$. These results together suggest that $x d h-1$ is the primary gene responsible for the observed abnormal cold tolerance phenotype.

$x d h-1$ encodes the $C$. elegans homolog of human xanthine dehydrogenase (XDH) (47\% homologous) (Figures S2A and B), and XDH itself contains iron-sulfur clusters, FAD, and molybdopterin domains (Figures S2A-C). FAD is an NAD binding site, molybdopterin is a redox center, and $\mathrm{XDH}$ xanthine dehydrogenase in dimer form catalyzes the hydroxylation of xanthine as well as its subsequent conversion to uric acid (Figure S2C). Iron-sulfur cluster domains are heavily conserved throughout the animal kingdom (Figures S2A and B), and our $x d h$-1(chrl) mutants possessed two point-mutations in this domain: one in a conserved splicing acceptor site and another in a non-conserved amino acid residue (Figures 1D and S2B). Yet another allele, ok3234, contains a deletion mutation at an NAD binding site (Figures 1D and S2B), and we used this allele to conduct the following analysis.

Cells expressing XDH-1 located in the AVJ and AIN head nerves, the intestine, and excretory cells were analyzed by fluorescent protein expression driven by the $x d h-1$ promoter (Figures 1F-I and S2D-F). To identify the essential tissue(s) responsible for $x d h$-1-dependent cold tolerance, we then expressed $x d h-1$ cDNA in specific tissues (Figure $1 \mathrm{~J}$ ) and found that $\mathrm{XDH}-1$ expression in nearly all neurons restored abnormal cold tolerance (Figure 1J; xdh-1;Ex[unc-14p::xdh-1cDNA]). xdh-1cDNA expression in intestine and excretory cells, however, did not rescue cold tolerance. This suggests neuronal XDH-1-activity is sufficient to maintain cold tolerance in C. elegans. 
Takagaki et al.

To determine the neuron type required for $x d h$-1-dependent cold tolerance, we performed a series of cell-specific rescue experiments by expressing $x d h$-1cDNA driven by various promoters (Figure 2). We found that abnormal $x d h-1$ mutant cold tolerance was rescued by expressing $x d h-1 c D N A$ in multiple neurons, including the AIN and AVJ interneurons (Figure 2). Moreover, expression of $x d h-1 c D N A$ in AIN and AVJ interneurons simultaneously using inx-17 and $h l h-34$ promoters rescued cold tolerance in $x d h-1$ mutants (Figure $2 \mathrm{~B}$ ). However, expression of $\mathrm{XDH}-1$ in either AIN or AVJ alone did not rescue normal function (Figure 2B). These results suggest that XDH-1 expressed in both AIN and AVJ are required for cold tolerance.

Since AIN and AVJ are interneurons that receive a variety of sensory information, we hypothesized that temperature sensation by any upstream sensory neuron may affect the activity of AIN and/or AVJ. There are nine such neurons, and five are known to mechanoreceptor-expressing sensory neuron. To determine whether these mechanoreceptor neurons are involved in cold tolerance, we tested the cold tolerance of mutants defective in various aspects of mechano-transduction. Experimentation showed that mutation to any of a number of mechanoreceptor components could lead to abnormal cold tolerance (Figure 3A), but mutation to deg-1, which encodes a monomeric Degenerin/Epithelial Sodium Channel (DEG/ENaC)-type mechanoreceptor, resulted in particularly severe cold tolerance dysfunction (Figure 3A).

ASG is the sole sensory neuron pair upstream of AIN/AVJ that expresses DEG-1, and is located in the head of C. elegans. $\mathrm{Ca}^{2+}$ imaging revealed that wild-type ASG responds to temperature changes by increasing intracellular $\mathrm{Ca}^{2+}$ concentration 
Takagaki et al.

(Figures $4 \mathrm{~A}$ and $\mathrm{B}$ ). In contrast, such a thermal response in deg-1 mutants was lower than in wild-type, which was rescued by ASG-specific expression of $\operatorname{deg}$-1cDNA (Figures 4A and B). These results suggest that DEG-1 is involved in the ASG sensory neuron temperature change response.

To investigate whether defective temperature sensation of ASG in deg-1 mutants causes abnormal neuronal activity in its downstream interneurons AIN and AVJ, we performed calcium imaging using cameleon calcium indicator. Mutants deg-1 AIN and AVJ calcium concentrations varied abnormally with thermal stimulus compared to wild-type (Figures 3B-E). In deg-1 mutants, AIN activity diminished while AVJ activity increased (Figures 3B-E), suggesting that AIN and AVJ are both required for normal cold tolerance and that their actions oppose one another. Moreover, the responsiveness of $x d h-1$ mutants AIN and AVJ were remarkably similar to the abnormal neural activities of these neurons in deg-1 mutant, indicating that the neural circuit from ASG to AIN and AVJ neurons regulates cold tolerance. These results are consistent with a result of genetic epistasis between $x d h-1$ and $\operatorname{deg}-1$ mutations that $d e g-1 ; x d h-1$ double mutants exhibited phenotypes comparable to either single mutation (Figure 3F), suggesting that $x d h-1$ and $d e g-1$ act within the same pathway.

To determine whether mechanoreceptor DEG-1 is involved in temperature sensation, we ectopically expressed DEG-1 in the non-thermosensitive ASE gustatory neuron then measured the resulting intracellular calcium dynamics. ASE gustatory neurons ectopically expressing DEG-1 strongly responded to changes in temperature, while wild-type ASE did not (Figures 4C and D). These results suggest that ectopic 
Takagaki et al.

expression of DEG-1 is sufficient to confer temperature responsiveness to the ASE gustatory neuron.

Next, we conducted electrophysiological experiments to determine the thermosensitivity of DEG-1 by applying the two-electrode voltage clamp recording method to Xenopus oocytes (Figures 4E-H). The DEG-1 mechanoreceptor and its mammalian homologue MDEG were expressed separately in Xenopus oocytes by injecting corresponding cRNAs for deg-1cDNA and MDEGcDNA. Thermal stimulus evoked internally-directed current in oocytes injected with deg-1cRNA and MDEGcRNA (Figures 4E-F, S4C and D), while control oocytes underwent no such changes (Figures 4E and S4C). These results suggest that DEG-1 and MDEG act as temperature-sensitive channels (Figures $4 \mathrm{G}$ and S4E; temperature threshold: $32.0 \pm 0.8^{\circ} \mathrm{C}$ for DEG-1 $(\mathrm{N}=8)$ and $31.0 \pm 0.3{ }^{\circ} \mathrm{C}$ for MDEG $\left.(\mathrm{N}=8)\right)$. It should be noted that the nematode vital temperature is between 13 and $27^{\circ} \mathrm{C}$, while the Xenopus oocyte DEG-1 reactions took place at $32^{\circ} \mathrm{C}$. Since the cold tolerant status in the worm body is constructed during the ambient cultivation condition such as $15{ }^{\circ} \mathrm{C}$ or $25{ }^{\circ} \mathrm{C}$ that is non-cold condition, as previously reported (Ohta et al., 2014), although we observed cold tolerance. Then, this is consistent with the result in this study that DEG-1 plays a role in temperature receptor sensing ambient temperature and in non-cold sensor.

Overall, the experiments performed in this study suggest that DEG/ENaC-type mechanoreceptor DEG-1 acts as a temperature sensor in ASG sensory neuron, which regulate AIN and AVJ interneurons to accomplish cold tolerance (Figure 4I). 
Takagaki et al.

\section{Materials and Methods}

\section{Strains}

We used the following C. elegans strains: N2 Bristol England (as wild-type) in all experiments, KHR066/RB2575 flp-17(ok3587) / $x d h-1(c h r 1), \quad$ KHR067/RB2379 xdh-1/F55B11.1(ok3234), VC883 tag-273(gk371), FX07280 / tbc-9(tm7280), KHR069 xdh-1(chr1), CB1066 mec-1(e1066), CB75 mec-2(e75), CB1338 mec-3(e1338), CB1339 mec-4(e1339), CB1340 mec-5(e1340), CB1472 mec-6(e1342), CB2477 mec-7(e1343), CB398 mec-8(e398), CB1515 mec-10(e1515), CB3284 mec-12(e1605), TU55 mec-14(u55), TU75 mec-15(u75), TU265 mec-17(u265), TU228 mec-18(u228), TU38 deg-1(u38), NC279 del-1(ok150), DH246 let-2(b246), VC1812 tab-1(gk858), and MT1098 unc-105(n506). See supplemental material for further details.

\section{Cold-tolerance Assay}

The cold-tolerance assay was performed according to previous reports (Ohta et al., 2014, Sonoda et al., 2016, Ujisawa et al., 2018, Ujisawa, Ohta et al., 2014). For this assay, we placed well-fed adult worms onto nematode growth medium (NGM) with $2 \%$ (w/v) agar, then seeded the medium with Escherichia coli OP50 once the worms began laying eggs. Adults were removed after $16-24 \mathrm{~h}$ at $15^{\circ} \mathrm{C}$, and progeny were left to mature for $120-130 \mathrm{~h}$ at $15^{\circ} \mathrm{C}$. Before the next generation had hatched, plates containing fresh adult worms were counted after being placed on ice for 20 minutes followed by transfer to a $2{ }^{\circ} \mathrm{C}$ refrigerated cabinet (CRB-41A Hitachi, Japan) for 48-96 h. Temperature inside the 
Takagaki et al.

refrigeration cabinet was monitored by both a digital and mercury thermometer. After the cold stimulus, plates were either repeatedly transferred to a room-temperature environment (total time over 3 hours) or stored at $15^{\circ} \mathrm{C}$ overnight. We then counted living and dead worms to calculate survival rates.

\section{Statistical analysis}

Cold tolerance testing was conducted on 6 or more plates for 3 or more non-consecutive days. All error bars indicate standard error of the mean (SEM). All statistical analyses assume normal distribution and were performed using parametric tests, the Tukey-Kramer method, Dunnett's test, or the unpaired t test (Welch). Multiple comparisons were performed using one-way ANOVA tested using the Tukey-Kramer method and Dunnett's test. Dunnett's test was performed to compare the left-most bar graph groups with other groups. Comparison between other group pairs was performed using the unpaired t test (Welch). ${ }^{*} \mathrm{p}<0.05 ; * * \mathrm{p}<0.01$.

\section{Molecular biology}

pNTN020 $x d h-1 p:: x d h-1$ genomic gene::gfp contains the $x d h-1$ full-length gene and a 3,346 bp segment upstream of $x d h-1$ amplified from the wild-type genome by PCR. GFP was inserted into the $x d h-1$ full-length gene, excluding stop codon. pNTN026 $x d h-1 p:: g f p$ contains the 3,346 bp upstream promoter sequence and the 3'-UTR of $x d h-1$ amplified by PCR from pNTN020. GFP was then inserted by pPDF95.75. The $x d h-1 p(1772 b p):: x d h-1 c D N A: \because g f p$ fragment contains the 1,772 bp upstream promoter 
sequence, the $x d h-1$ gene, and $x d h-1 c D N A$ amplified by PCR from pNTN058 (supplemental methods), which was used for the transgenic expression experiment. pNTN118 $x d h-1 p:: d s R e d m$ contains the $x d h-1$ promoter and dsRedm.

pNTN027 contains a Kozak sequence, the $x d h 1$ cDNA that was amplified by PCR from the cDNA library, and the 3'-UTR of the unc-54 gene. The promoter sequences, unc-14p (1.4 kb), pgp-12p (3.5 kb), ges-1p (3.3 kb), xdh-1p (3.4 kb), dat-1p (0.7 kb), osm-6p (2 kb), ncs-1p (3.1 kb), glr-1p (5.4 kb), unc-8p (4.2 kb), unc-47p (0.3 kb), acr-2p (3.4 kb), eat-4p (6.4 kb), unc-42p (3 kb), unc-86p (3.6 kb), ocr-4p (4.8 kb), ceh-10p (3.5 kb), sra-6p (3.8 kb), lim-4p (3.6 kb), ser-2p (4.1 kb), inx-17p (1.2 kb), and $h l h-34 p(2.5 \mathrm{~kb})$ were inserted upstream of pNTN027 $x d h-1 c D N A$, to create respectively, pNTN034, 035, 036, 046, 047, 048, 049, 050, 051, 052, 053, 054, 055, 057, 059, 060, 061, 063, 064, 067, and 068 plasmids for cellular experimentation. pNTN075 hlh-34p::yc3.60 contains the $2.5 \mathrm{~kb} h l h-34 p$ gene and the $y c 3.60$ gene. pNTN106 gcy-5p::deg-1cDNA contains the gcy-5 promoter received from Dr. Iino. pNTN116 contains the inx-17 promoter, the $y c 3.60$ gene, and the 3'-UTR of the let-858 gene. pNTN123 gcy-21p::yc3.60 contains the 1,403 bp upstream promoter sequence of the gcy-21 gene amplified by PCR from the wild-type genome, which was created by replacing the $h l h-34 p$ gene of pNTN075 with gcy-21p. Previous reports described the gcy-21p::GFP construct containing the $1^{\text {st }}$ and $2^{\text {nd }}$ exons and $1^{\text {st }}$ intron as inducing expression of GFP strongly in ASG and weakly in ADL. However, the gcy-21p::GFP construct excluding all exons and introns induces GFP expression in ASG only. We therefore used $g c y-21 p$ as an ASG-specific promoter. pNTN126 gcy-21p::deg-1cDNA 
Takagaki et al.

contains the 1,403 bp upstream promoter sequence of the $g c y-21$ and the deg-1cDNA. pMIU34 flp-6p::CeG-CaMP8 contains a 2,680 bp upstream promoter sequence for the flp-6 gene (G-CaMP8) and is codon-optimized for C. elegans (CeG-CaMP8). deg-1cDNA was inserted into a pGEMHE vector containing Xenopus beta-globin 5' and 3' UTR for electrophysiological recording (pNTN119).

\section{Two-electrode voltage clamp recording in Xenopus oocytes}

deg-1cRNA and MDEGcRNA were separately injected into oocytes and incubated at $18^{\circ} \mathrm{C}$ for 3-6 days before electrophysiological recordings were made. We held membrane potential at $-80 \mathrm{mV}$ and recorded macroscopic current using the two-electrode voltage-clamp technique with a bath clamp amplifier (OC-725C; Warner Instruments, USA) and pClamp software (Molecular Devices, USA) in bath solution containing $100 \mathrm{mM} \mathrm{NaCl}, 2 \mathrm{mM} \mathrm{MgCl}$, and $10 \mathrm{mM}$ HEPES (pH 7.3). Temperature stimulation was regulated using a lab-made temperature controller with a range of 10 to $35^{\circ} \mathrm{C}$ and was monitored by both a thermistor prove adjacent to the oocytes and a thermometer (DIGITAL THERMOMETER PTC-401; UNIQUE MEDICAL, Japan). Arrhenius plot indicates the current amplitude induced by temperature changes on the $\mathrm{y}$-axis (log scale) versus the inverse of temperature on the $\mathrm{x}$-axis $(1000 / \mathrm{K})$. Temperature thresholds were determined by the intersection of the two liner regions (magenta lines), and all thresholds were then averaged. As a negative control experiment, we used amiloride, an inhibitor of sodium ion channel, and oocytes were incubated at $18^{\circ} \mathrm{C}$ in bath's solution with $500 \mu \mathrm{M}$ amiloride (Sigma-Aldrich) for $48 \mathrm{hr}$. 
Takagaki et al.

\section{In vivo Calcium Imaging}

In vivo calcium imaging was performed according to previous reports (Kuhara, Ohnishi et al., 2011, Ohta et al., 2014, Ujisawa et al., 2018). We used yellow cameleon (yc3.60) and GCaMP8 as genetically encodable calcium indicators. When using GCaMP8, we co-expressed tag-RFP (pKOB006 gcy-5p::tag-RFP) in order to measure the fluorescence ratio between GCaMP and tagRFP(Kobayashi, Nakano et al., 2016). Worms expressing the calcium indicator in one or more neurons were glued to a $2 \%$ (w/v) agar pad on glass, immersed in M9 buffer, and covered by a cover glass. Fluorescence of cyan (CFP) and yellow (YFP) by YC3.60, or green (GCaMP8) and red (tag-RFP), was simultaneously captured using a EM-CCD camera EVOLVE512 (photometrics, USA). Changes in intracellular calcium concentrations were measured as the yellow/cyan fluorescence ratio for YC3.60 or green/red fluorescence ratio for GCaMP8. See supplemental experimental material for more detail.

Acknowledgments: We thank I. Mori, H. R. Horvitz, C. I. Bargmann, D. Yan, Y. Jin, K. Ashrafi, D. H. Hall, L. Bianchi, Y. Iino, T. Nakatani, T. Ii, J. Burkhead, J. M. Kaplan, T. G. Kusakabe and S. Mitani for sharing DNA constructs and strains; the National Bioresource Project (Japan) and the Caenorhabditis Genetic Center for strains; T. Miura and K. Kanai for supporting phenotypic experiments or maintenance of experimental systems; and members of the Kuhara Laboratory for comments and stimulating discussion. We thank Mr. Eric Odle for English editing and proofreading of the 
Takagaki et al.

manuscript. We would like to thank the staff of Comparative Genomics Laboratory at NIG for supporting genome sequencing. Funding: A.K. was supported by the Asahi Glass Foundation, the Takeda Science Foundation, the Naito Foundation, the Hirao Taro Foundation of KONAN GAKUEN for Academic Research, AMED Mechano Biology (19gm5810024h0003), JSPS KAKENHI (15K21744, 17K19410, 18H02484); and KAKENHI (15H05928, 16H06279) from MEXT Japan. A.O. was supported by the Daiichi Sankyo Foundation of Life Science, the Takeda Science Foundation, the Naito Foundation, and JSPS KAKENHI (16J00123, 18K06344). N.T. was supported by JSPS KAKENHI (18J10116). Y.F. was supported by KAKENHI (18H04697) from MEXT Japan. Computations were partially performed on the NIG supercomputer at ROIS National Institute of Genetics.

Author contributions: N.T., A.O., K.O., Y.M., A.T., Y.F. and A.K. performed the experiments; N.T., A.O., A.T., Y.F. and A.K. designed the experiments, interpreted the results, and wrote the final report. Competing interests: The authors declare no competing interests. Data and material availability: All data required to evaluate study conclusions can be found in either the main text or supplemental materials. Requests for further information should be addressed to A.O. or A.K. Information regarding data, figures, or other research findings may be addressed to the corresponding authors. 


\section{References}

Askwith CC, Benson CJ, Welsh MJ, Snyder PM (2001) DEG/ENaC ion channels involved in sensory transduction are modulated by cold temperature. Proc Nat1 Acad Sci USA 98: 6459-63

Barr MM (2003) Super models. Physiol Genomics 13: 15-24

Brenner S (1974) The genetics of Caenorhabditis elegans. Genetics 77: 71-94

Chalfie M, Wolinsky E (1990) The identification and suppression of inherited neurodegeneration in Caenorhabditis elegans. Nature 345: 410-6

Chandrashekar J, Kuhn C, Oka Y, Yarmolinsky DA, Hummler E, Ryba NJ, Zuker CS (2010) The cells and peripheral representation of sodium taste in mice. Nature 464: 297-301

Chen Y, Bharill S, O'Hagan R, Isacoff EY, Chalfie M (2016) MEC-10 and MEC-19 Reduce the Neurotoxicity of the MEC-4(d) DEG/ENaC Channel in Caenorhabditis elegans. G3 (Bethesda, Md) 6: 1121-30

Chung HY, Baek BS, Song SH, Kim MS, Huh JI, Shim KH, Kim KW, Lee KH (1997) Xanthine dehydrogenase/xanthine oxidase and oxidative stress. Age 20: $127-40$

Dhaka A, Viswanath V, Patapoutian A (2006) Trp ion channels and temperature sensation. Annu Rev Neurosci 29: 135-61

Geffeney SL, Cueva JG, Glauser DA, Doll JC, Lee TH, Montoya M, Karania S, Garakani AM, Pruitt BL, Goodman MB (2011) DEG/ENaC but not TRP channels are the major mechanoelectrical transduction channels in a $\mathrm{C}$. elegans nociceptor. Neuron 71: 845-57

Hall DH, Gu G, Garcia-Anoveros J, Gong L, Chalfie M, Driscoll M (1997) Neuropathology of degenerative cell death in Caenorhabditis elegans. The Journal of neuroscience : the official journal of the Society for Neuroscience 17: $1033-45$

Kobayashi K, Nakano S, Amano M, Tsuboi D, Nishioka T, Ikeda S, Yokoyama G, Kaibuchi K, Mori I (2016) Single-Cell Memory Regulates a Neural Circuit for Sensory Behavior. Cell Rep 14: 11-21

Kuhara A, Ohnishi N, Shimowada T, Mori I (2011) Neural coding in a single sensory neuron controlling opposite seeking behaviours in Caenorhabditis 
Takagaki et al.

elegans. Nat Commun 2: 355

Liu L, Leonard AS, Motto DG, Feller MA, Price MP, Johnson WA, Welsh MJ (2003) Contribution of Drosophila DEG/ENaC genes to salt taste. Neuron 39: $133-46$

Murray P, Hayward SA, Govan GG, Gracey AY, Cossins AR (2007) An explicit test of the phospholipid saturation hypothesis of acquired cold tolerance in Caenorhabditis elegans. Proc Natl Acad Sci US A 104: 5489-94

Ohta A, Kuhara A (2013) Molecular mechanism for trimeric G protein-coupled thermosensation and synaptic regulation in the temperature response circuit of Caenorhabditis elegans. Neurosci Res 76: 119-24

Ohta A, Ujisawa T, Sonoda S, Kuhara A (2014) Light and pheromone-sensing neurons regulates cold habituation through insulin signalling in Caenorhabditis elegans. Nature communications 5: 4412

Okahata M, Ohta A, Mizutani H, Minakuchi Y, Toyoda A, Kuhara A (2016) Natural variations of cold tolerance and temperature acclimation in Caenorhabditis elegans. J Comp Physiol B 186: 985-998

Saidak Z, Louandre C, Dahmani S, Sauzay C, Guedda S, Chauffert B, Chatelain D, Ceballos-Picot I, Galmiche A (2018) A pan-cancer study of the transcriptional regulation of uricogenesis in human tumours: pathological and pharmacological correlates. Bioscience reports 38

Saksela M, Lapatto R, Raivio KO (1999) Irreversible conversion of xanthine dehydrogenase into xanthine oxidase by a mitochondrial protease. FEBS Lett 443: 117-20

Shen WL, Kwon Y, Adegbola AA, Luo J, Chess A, Montell C (2011) Function of rhodopsin in temperature discrimination in Drosophila. Science 331: 1333-6

Sonoda S, Ohta A, Maruo A, Ujisawa T, Kuhara A (2016) Sperm Affects Head Sensory Neuron in Temperature Tolerance of Caenorhabditis elegans. Cell Rep 16: 56-65

Takeishi A, Yu YV, Hapiak VM, Bell HW, O'Leary T, Sengupta P (2016) Receptor-type Guanylyl Cyclases Confer Thermosensory Responses in C. elegans. Neuron 90: 235-44

Terawaki H, Murase T, Nakajima A, Aoyagi K, Fukushima N, Tani Y, 
Takagaki et al.

Nakamura T, Kazama JJ (2017) The Relationship between Xanthine Oxidoreductase and Xanthine Oxidase Activities in Plasma and Kidney Dysfunction. Journal of Clinical \& Experimental Nephrology 02

Ugawa S, Yamamoto T, Ueda T, Ishida Y, Inagaki A, Nishigaki M, Shimada S (2003) Amiloride-insensitive currents of the acid-sensing ion channel-2a (ASIC2a)/ASIC2b heteromeric sour-taste receptor channel. The Journal of neuroscience : the official journal of the Society for Neuroscience 23: 3616-22 Ujisawa T, Ohta A, Ii T, Minakuchi Y, Toyoda A, Ii M, Kuhara A (2018) Endoribonuclease ENDU-2 regulates multiple traits including cold tolerance via cell autonomous and nonautonomous controls in Caenorhabditis elegans.

\section{Proc Natl Acad Sci USA}

Ujisawa T, Ohta A, M. O, S. S, Kuhara A (2014) Cold tolerance assay for studying cultivation-temperature-dependent cold habituation in C. elegans. Protocol Exchange

Waldmann R, Champigny G, Voilley N, Lauritzen I, Lazdunski M (1996) The mammalian degenerin MDEG, an amiloride-sensitive cation channel activated by mutations causing neurodegeneration in Caenorhabditis elegans. J Biol Chem 271: 10433-6

Wang Y, Apicella A, Jr., Lee SK, Ezcurra M, Slone RD, Goldmit M, Schafer WR, Shaham S, Driscoll M, Bianchi L (2008) A glial DEG/ENaC channel functions with neuronal channel DEG-1 to mediate specific sensory functions in C. elegans. The EMBO journal 27: 2388-99

Wemmie JA, Askwith CC, Lamani E, Cassell MD, Freeman JH, Jr., Welsh MJ (2003) Acid-sensing ion channel 1 is localized in brain regions with high synaptic density and contributes to fear conditioning. The Journal of neuroscience : the official journal of the Society for Neuroscience 23: 5496-502

Wemmie JA, Chen J, Askwith CC, Hruska-Hageman AM, Price MP, Nolan BC, Yoder PG, Lamani E, Hoshi T, Freeman JH, Jr., Welsh MJ (2002) The acid-activated ion channel ASIC contributes to synaptic plasticity, learning, and memory. Neuron 34: 463-77

Wemmie JA, Coryell MW, Askwith CC, Lamani E, Leonard AS, Sigmund CD, Welsh MJ (2004) Overexpression of acid-sensing ion channel 1a in transgenic 
Takagaki et al.

mice increases acquired fear-related behavior. Proc Natl Acad Sci US A 101: 3621-6

Xi H, Schneider BL, Reitzer L (2000) Purine catabolism in Escherichia coli and function of xanthine dehydrogenase in purine salvage. Journal of bacteriology 182: 5332-41

Zha XM, Wemmie JA, Green SH, Welsh MJ (2006) Acid-sensing ion channel $1 \mathrm{a}$ is a postsynaptic proton receptor that affects the density of dendritic spines. Proc Natl Acad Sci US A 103: 16556-61

Zhong L, Hwang RY, Tracey WD (2010) Pickpocket is a DEG/ENaC protein required for mechanical nociception in Drosophila larvae. Curr Biol 20: 429-34

Ziemann AE, Allen JE, Dahdaleh NS, Drebot, II, Coryell MW, Wunsch AM, Lynch CM, Faraci FM, Howard MA, 3rd, Welsh MJ, Wemmie JA (2009) The amygdala is a chemosensor that detects carbon dioxide and acidosis to elicit fear behavior. Cell 139: 1012-21

\section{Figure legends}

Figure 1. Neuronal $\mathrm{XDH}-1$ regulates cold tolerance. Animals grown at $15^{\circ} \mathrm{C}$ were transferred to $2^{\circ} \mathrm{C}$ for 48 hours. Error bar indicates SEM (B, C, J). (A) Schematic of $C$. elegans cold tolerance. $20^{\circ} \mathrm{C}$-cultivated worms do not survive at $2^{\circ} \mathrm{C}$, while $15^{\circ} \mathrm{C}$-cultivated worms do. (B) Cold tolerance when cultivated at $15^{\circ} \mathrm{C}$. chrl and $x d h-1$ mutants exhibit abnormal cold tolerance. Number of assays $\geq 10$. Comparisons were performed using Dunnett's test. **p $<0.01$. (C) Transgenic rescue of $x d h-1$ mutants with plasmid pNTN020 containing wild-type $x d h$ - 1 fused with GFP and native promoter sequence. Number of assays $\geq 11$. Comparisons were performed using the Tukey-Kramer method. $* * \mathrm{p}<0.01$. (D) Exons of $x d h-1$ gene are boxed and numbered. 
Takagaki et al.

Positions of chrl point mutations and ok3234 deletion mutation are shown. (E) $x d h-1$ encodes xanthine dehydrogenase. Shading represents different domains in XDH-1. The amino acid identity and similarity between $\mathrm{XDH}-1$ and human $\mathrm{XDH}$ are given for each domain. (F) Schematic diagram of expression pattern (green). (G) $x d h-1 p:: G F P$ expressions in neurons, intestine, and excretory cells. (H) Wild-type animals expressing $x d h-1 c D N A:: G F P$ driven by $x d h-1$ promoter and AVJ-specific expression of dsRedm (wild-type; Ex[xdh-1p::xdh-1cDNA::GFP, hlh-34p::dsRedm]). Arrow indicates white region that suggests co-expression of GFP and dsRedm in AVJ interneuron. XDH-1::GFP fluorescence was spotty, and white region was faint. (I) Wild-type animals expressing dsRedm driven by $x d h-1$ promoter and AIN-specific expression of YFP (wild-type; Ex[xdh-1p::dsRedm, inx-17p::YFP]). Arrow indicates white region that suggests co-expression of YFP and dsRedm in AIN interneuron. (J) Abnormal cold tolerance in $x d h-1$ mutant was rescued by expressing $x d h-1 c D N A$ in almost all neurons. Number of assays $\geq 9$. Comparisons were performed using the Tukey-Kramer method. $* * \mathrm{p}<0.01$. Data from wild-type and xdh-1; Ex[unc-14p::xdh-1cDNA] animals are shown in Fig. 2A.

Figure 2. Cell-specific rescue experiments for abnormal xdh-1 mutant cold tolerance. $(\mathrm{A}, \mathrm{B})$ Expression of $x d h-1 c D N A$ driven by various promoters. $15^{\circ} \mathrm{C}$-cultivated animals at adult stage were transferred to $2^{\circ} \mathrm{C}$. Irregular cold tolerance in $x d h-1$ mutants was rescued by expressing $x d h-1 c D N A$ in both AVJ and AIN interneurons. Number of assays $\geq 6$. Error bar indicates SEM. Comparisons were performed using the Tukey-Kramer 
Takagaki et al.

method. $* * p<0.01$. Data from wild-type and xdh-1; Ex[unc-14p::xdh-1cDNA] worms are shown in Fig. 1J.

Figure 3. AIN and AVJ interneurons are involved in the thermosensitivity. Calcium imaging was performed using yellow cameleon 3.60 (B to E). Comparisons were performed using the Tukey-Kramer method. **p $<0.01$. (B, D, F). (A) Cold tolerance of mechanoreceptor-related gene knock-out mutants. Animals grown at $15^{\circ} \mathrm{C}$ were transferred to $2^{\circ} \mathrm{C}$ for 96 hours. deg-1 mutants exhibited pronounced abnormality. Number of assays $\geq 10$. Error bar indicates SEM. Comparisons were performed using Dunnett's test. ${ }^{*} \mathrm{p}<0.05 ;{ }^{*} \mathrm{p}<0.01$. (B, C) AIN calcium imaging in wild-type, $x d h-1$ mutants and deg-1 mutants cultivated at $15^{\circ} \mathrm{C}$. Bar graph shows average change in cyan-yellow fluorescence ratio within 11 seconds from second 130 to 141 . Each graph represents average response to temperature stimulus. Number of assays $\geq 17$. Error bar indicates SEM. Bar graph color key is same as that of corresponding response curve in panel B (C). (D, E) AVJ calcium imaging in wild-type, $x d h-1$ mutants and deg-1 mutants cultivated at $15^{\circ} \mathrm{C}$. Bar graph, average change in cyan-yellow fluorescence ratio within seconds 11 from second 90 to 101 . Number of assays $\geq 17$. Error bar indicates SEM. Bar graph color key is same as that of corresponding response curve in panel D (E). (F) Cold tolerance assays for deg-1;xdh-1 double mutants. Number of assays $\geq 12$. Error bar indicates SEM.

Figure 4. DEG-1 is involved in cold tolerance and thermosensitivity. Calcium imaging 
Takagaki et al.

was performed using yellow cameleon 3.60 (A, B) and GCaMP8 with tag-RFP (C, D). Comparisons were performed using unpaired t test (Welch). ${ }^{*} \mathrm{p}<0.05 ; * * \mathrm{p}<0.01$ (D, H). (A, B) Calcium imaging of ASG in animals cultivated at $15^{\circ} \mathrm{C}$. Bar graph, average change in cyan-yellow fluorescence ratio within 11 seconds from second 230 to 241 . Number of assays $\geq 20$. Error bar indicates SEM. Previously reported, wild-type deg-1 gene restored the $u 38$ abnormal touch sensitivity although the $u 38$ is a dominant negative mutation (Chalfie \& Wolinsky, 1990) Bar graph color key is same as that of corresponding response curve in panel A (B). Comparisons were performed using the Tukey-Kramer method. ${ }^{* *} \mathrm{p}<0.01$ (B). (C, D) Calcium imaging of non-thermosensitive ASE neurons in $15^{\circ} \mathrm{C}$-cultivated wild-type worms ectopically expressing DEG-1 in ASE. Bar graph shows average change in cyan-yellow fluorescence ratio within 11 seconds from second 230 to 241 . Number of assays $\geq 19$. Error bar indicates SEM. Bar graph color key is same as that of corresponding response curve in panel C (D). (E-H) Reactions (representative current traces) to thermal stimulus in Xenopus oocytes expressing DEG-1. (E) Heating phase relationship between current and temperature shown in panels F. Data from non-injected oocytes $(\mathrm{n}=8)$. (F) Representative current (upper) and temperature (lower) traces $(n=8)$. (G) Arrhenius plots from data in panels E. Data obtained from temperature increase is shown in panels F. Temperature threshold was determined by intersection of the two extended lines shown in magenta $(n=8)$. (H) Suppression of heat-evoked currents by incubating in bath's solution with amiloride, an inhibitor of sodium ion channel $(\mathrm{n} \geq 5$ ). (I) A model for neuronal circuit for cold tolerance modulated by the signaling from ASG sensory neuron to AIN and AVJ 
Takagaki et al.

interneuron. ASG senses temperature via DEG-1, connects to AIN (headed arrow). ASG indirectly connects to AVJ via unidentified neuron (dotted-line arrow). ASG-AIN-AVJ neural circuit positively regulates cold tolerance, in which ASG positively and negatively controls neuronal activity of AIN and AVJ respectively. 
bioRxiv preprint doi: https://doi.org/10.1101/673863; this version posted June 18, 2019. The copyright holder for this preprint (which was

Figure 1

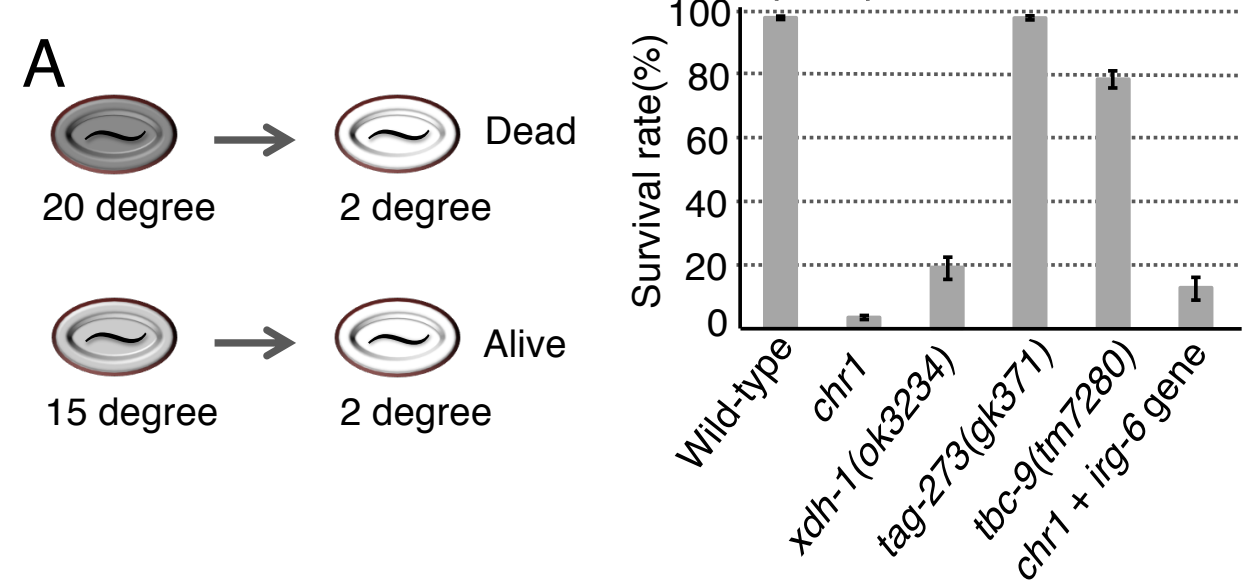

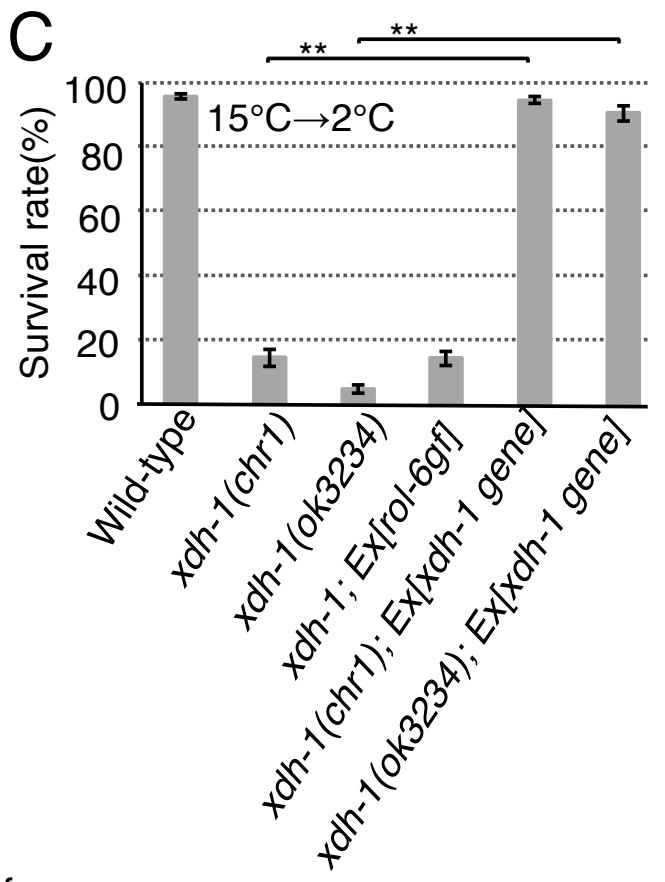

xdh-1 gene ok3234 deletion

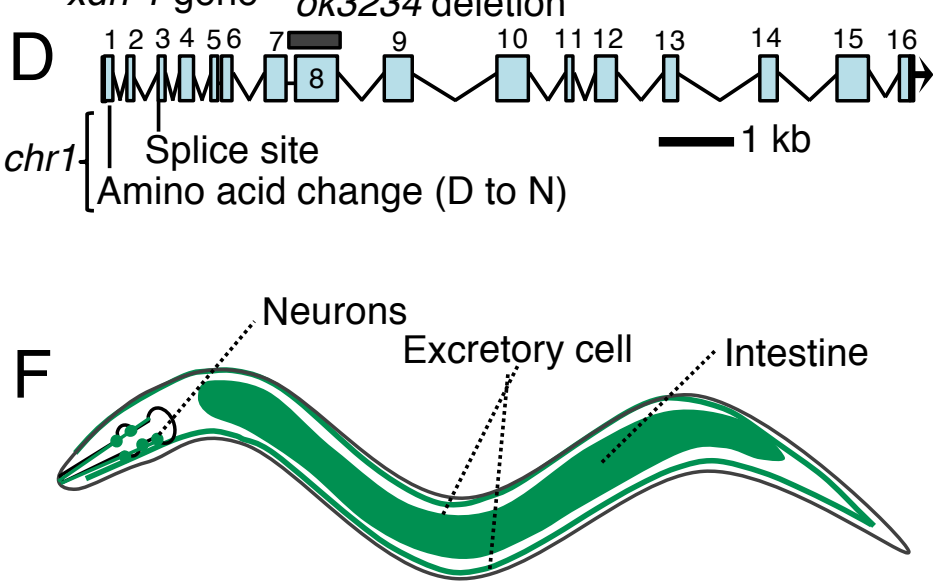

E

Iron-sulfur cluster

FAD

Molybdopterin

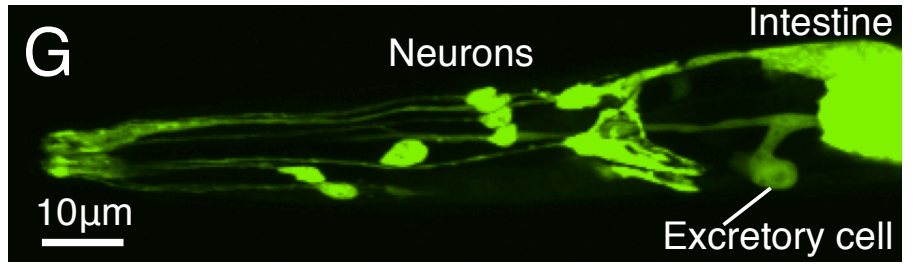

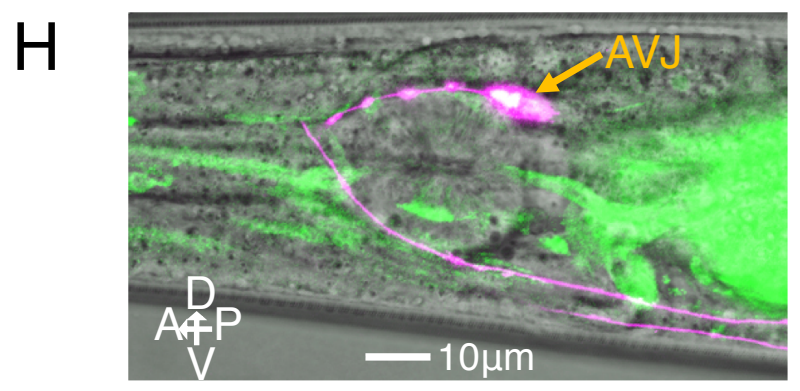
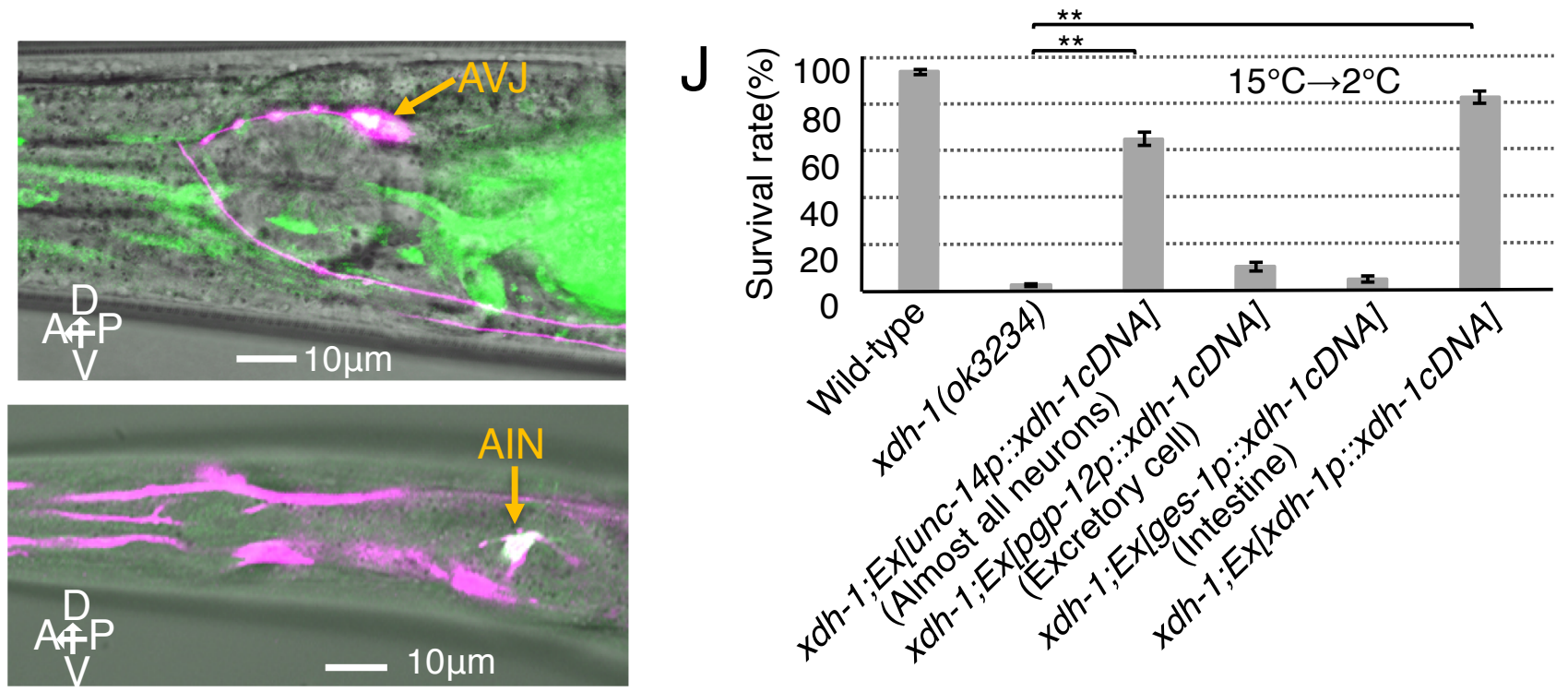

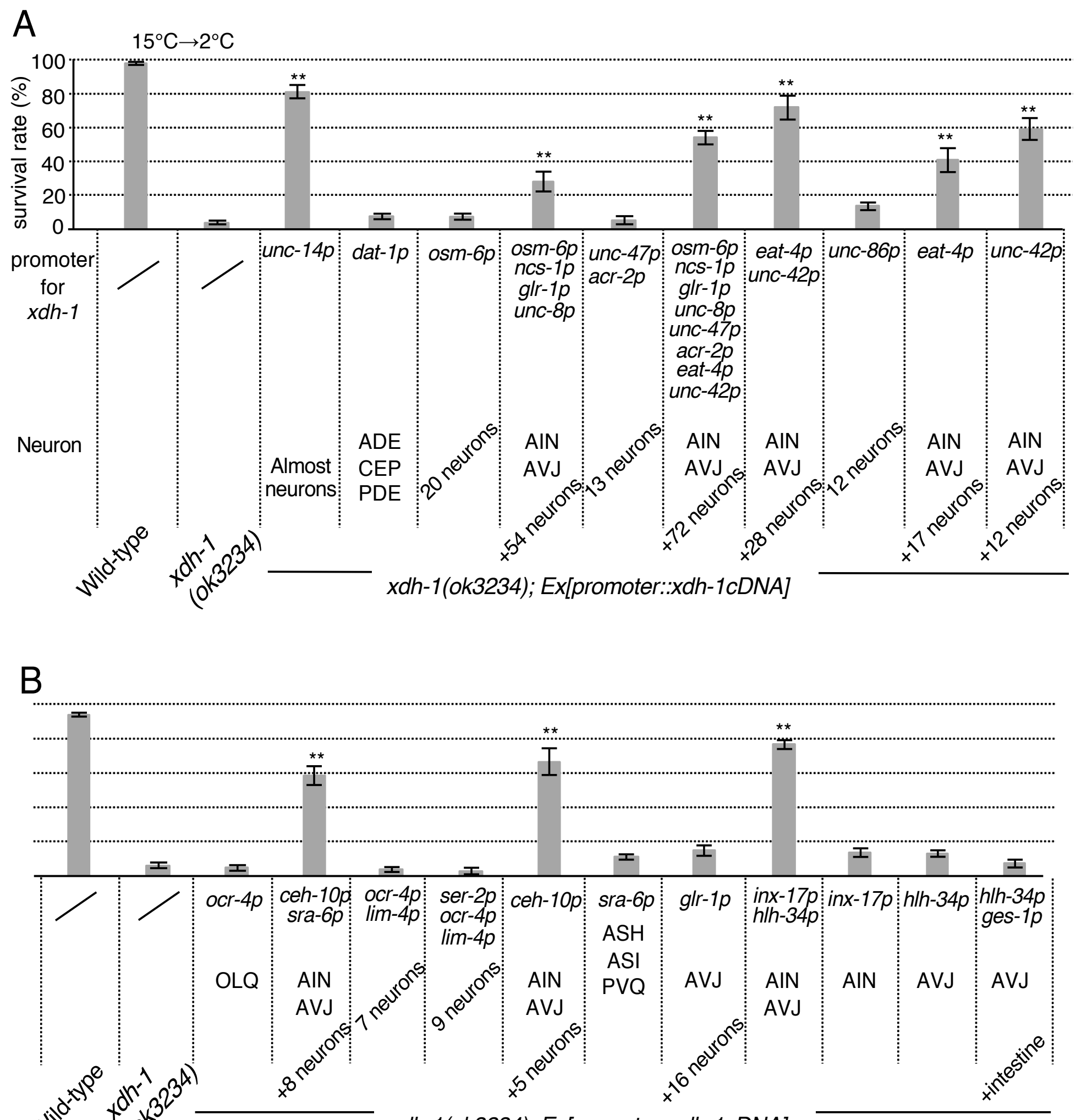

xdh-1(ok3234); Ex[promoter::xdh-1cDNA] 
bioRxiv preprint doi: https://doi.org/10.1101/673863; this version posted June 18, 2019. The copyright holder for this preprint (which was

Figure 3

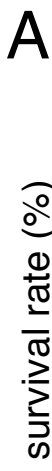

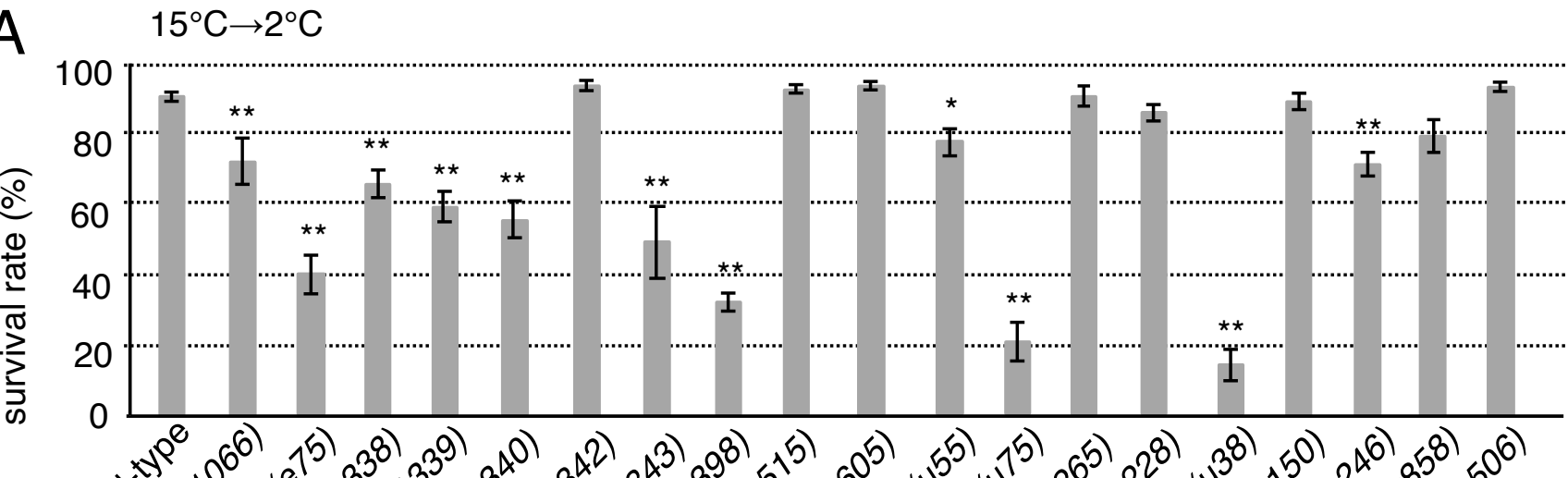

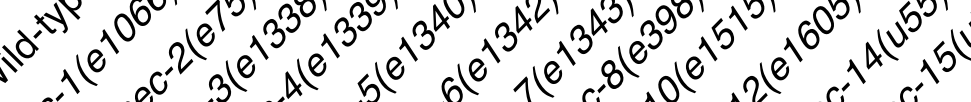

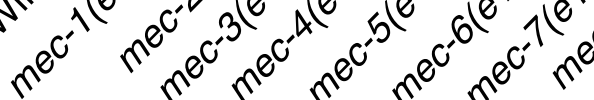
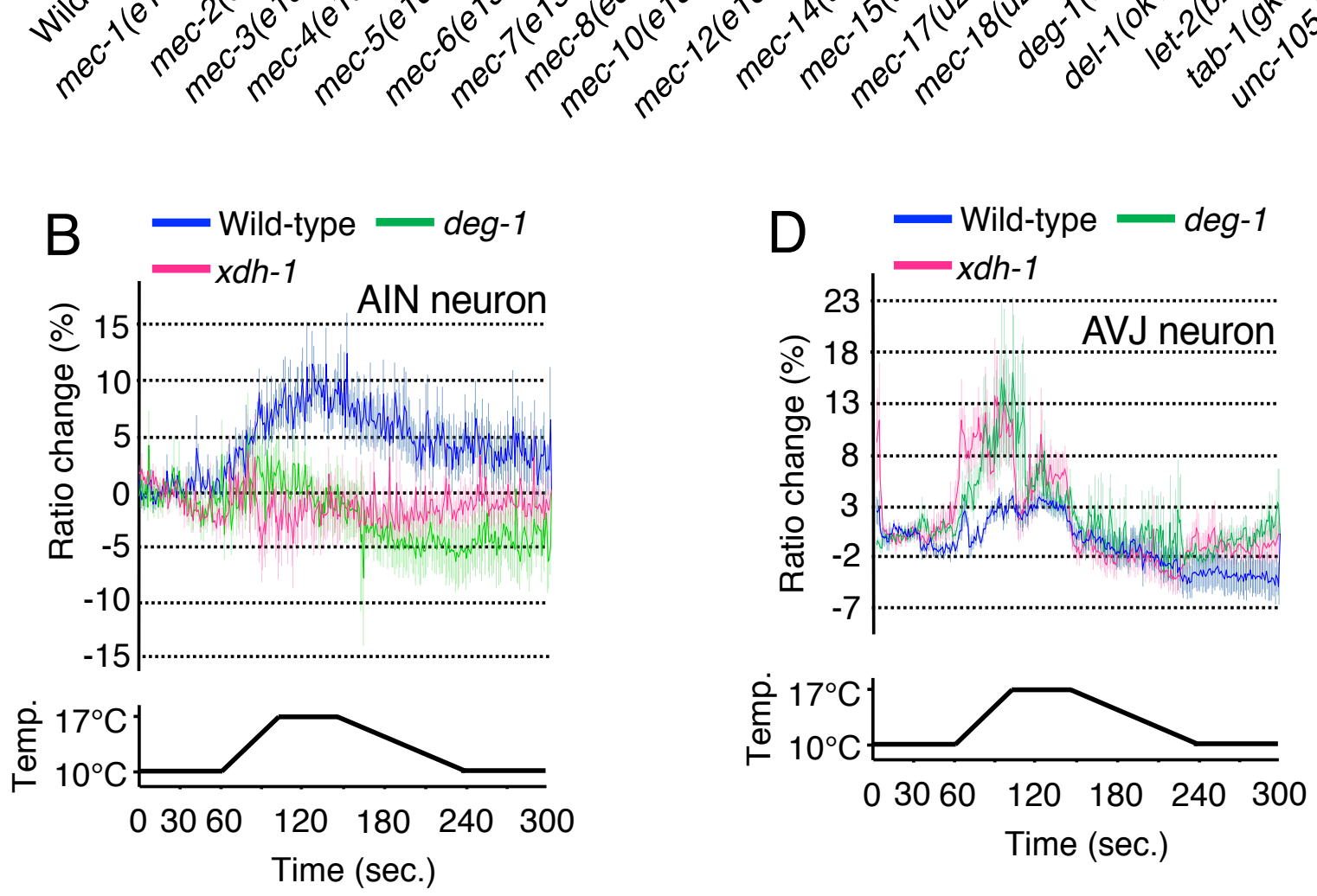

C
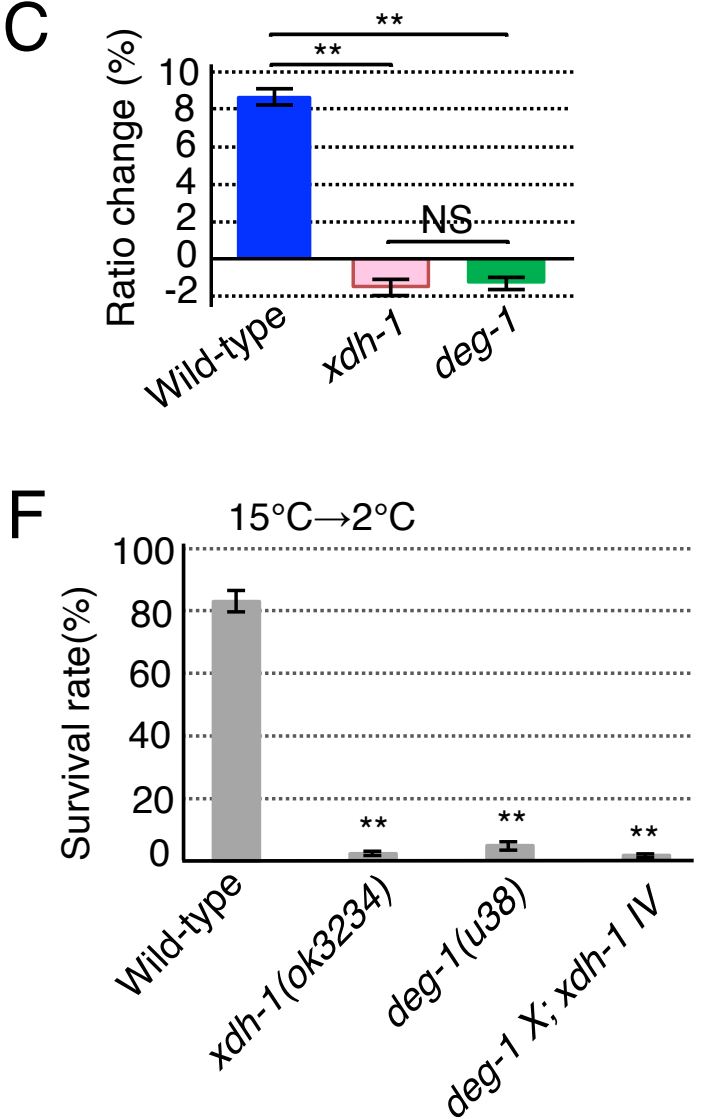

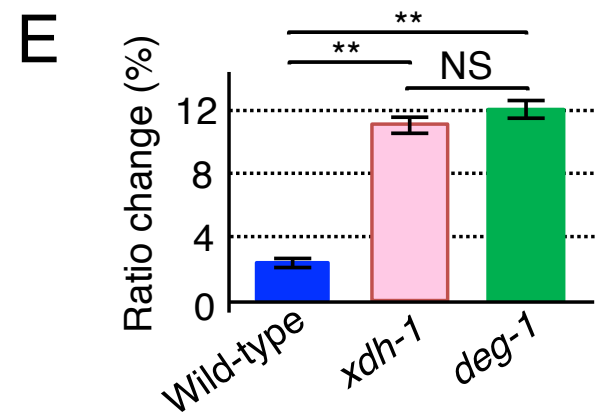


Figure 4
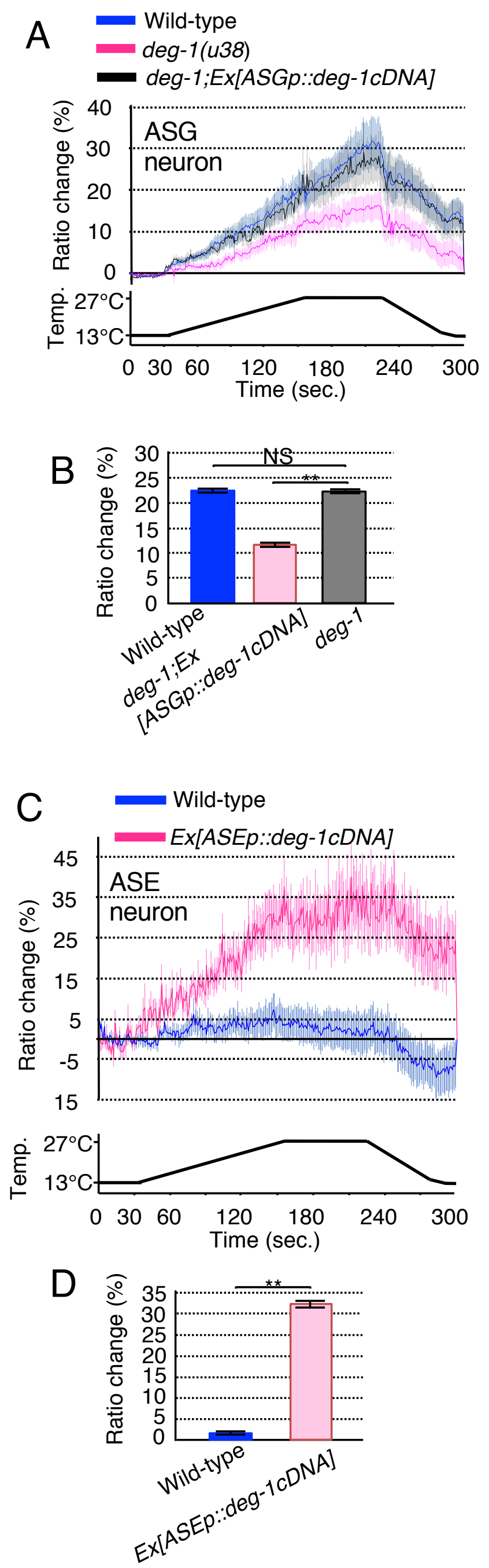
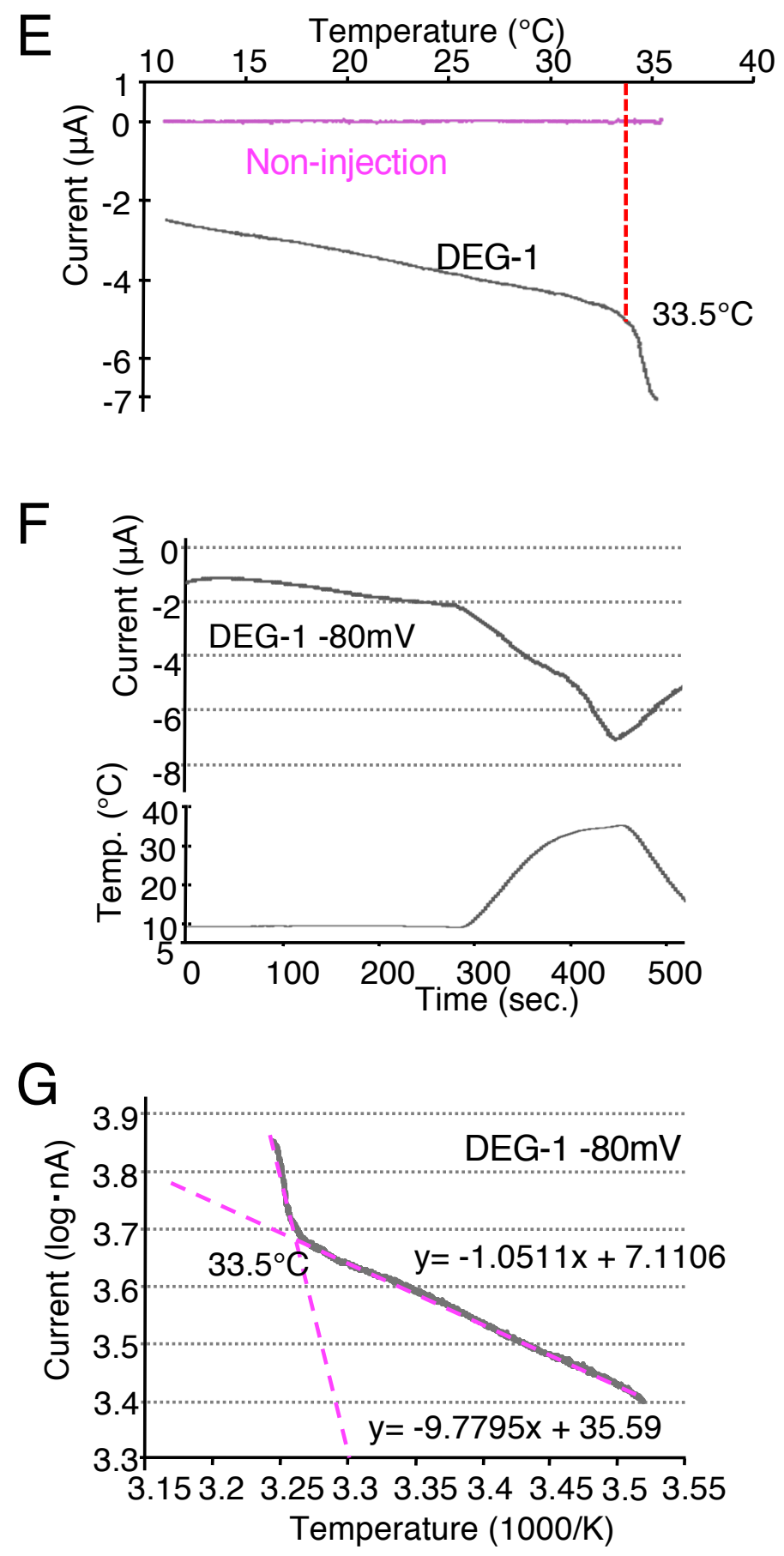

$\mathrm{H}$
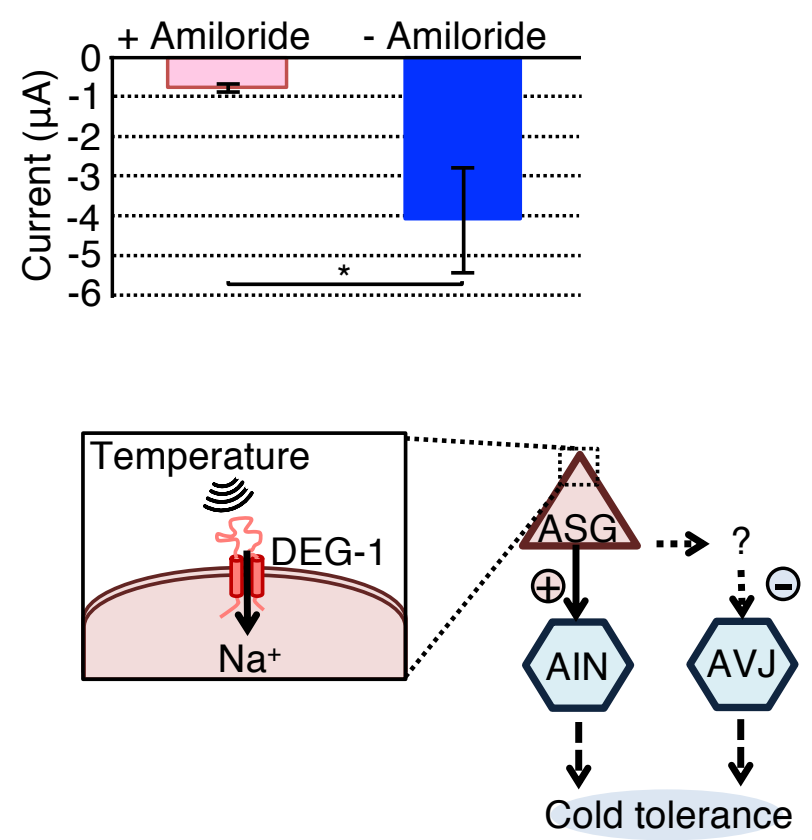


\section{Figure S1}

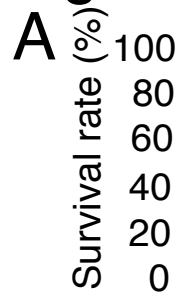

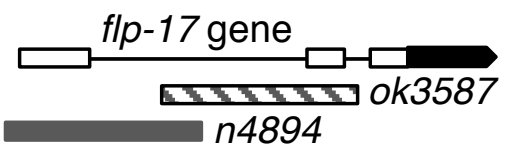

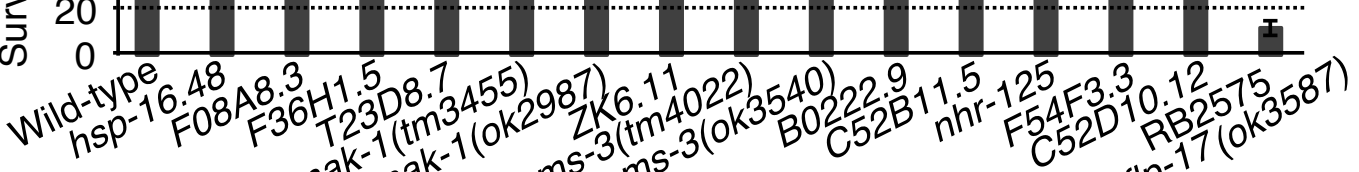

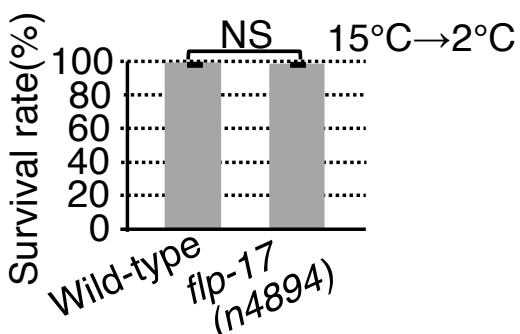

C

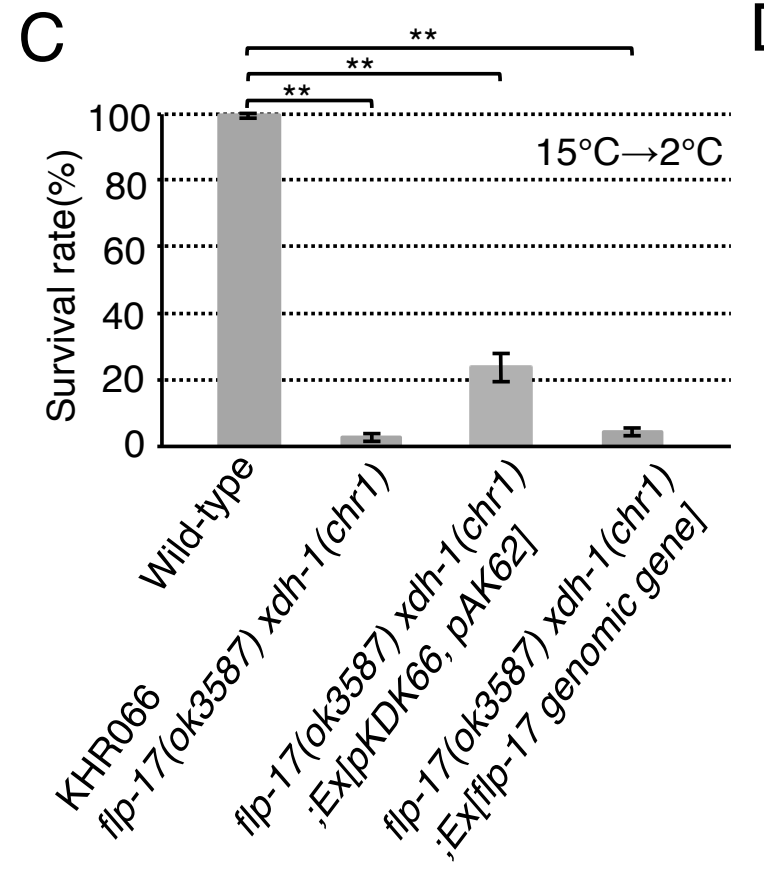

D

$f P^{-1}$

$(n 4894$

\begin{tabular}{|c|c|c|}
\hline & position & Abnormal phenotype strain genome pool \\
\hline \hline \multirow{3}{*}{ Chromosome I } & 4377261 & Wild-type \\
\cline { 2 - 3 } & 6290450 & Wild-type/RB2575 \\
\cline { 2 - 3 } & 14874142 & Wild-type/RB2575 \\
\hline \multirow{3}{*}{ Chromosome II } & 4795820 & Wild-type \\
\cline { 2 - 3 } & 6948145 & Wild-type \\
\hline \multirow{3}{*}{ Chromosome III } & 1540306 & Wild-type/RB2575 \\
\cline { 2 - 3 } & 592022 & \\
\hline \multirow{3}{*}{ Chromosome IV } & 8894988 & Wild-type/RB2575 \\
\cline { 2 - 3 } & 1981523 & Wild-type/RB2575 \\
\hline & 7663908 & Wild-type/RB2575 \\
\hline \multirow{3}{*}{ Chromosome V } & 263872775 & Wild-type/RB2575 \\
\cline { 2 - 3 } & 8773000 & RB2575 \\
\cline { 2 - 3 } & 17057384 & Wild-type/RB2575 \\
\hline \multirow{3}{*}{ Chromosome VI } & 2029668 & Wild-type/RB2575 \\
\cline { 2 - 3 } & 9299203 & Wild-type/RB2575 \\
\cline { 2 - 3 } & 13054947 & Wild-type/RB2575 \\
\hline
\end{tabular}

$E$

\begin{tabular}{|c|c|c|c|c|c|c|c|c|c|c|}
\hline & \multicolumn{10}{|c|}{ Genotype } \\
\hline $\mathrm{cM}$ & -12.45 & 3.38 & 3.96 & 4.83 & 6.13 & 6.46 & 9.30 & 11.87 & 12.18 & 16.24 \\
\hline Strain & & & & & & irg-6 & $t b c-9$ & F55B1.1 & $\operatorname{tag}-273$ & $f \mid p-17$ \\
\hline \#bc5-1 & Wild-type & WT/RB & WT/RB & WT/RB & Unkinowi & Unknnown & Unknọow & RB2575 & RB2575 & RB2575 \\
\hline \#bc5-2 & Wild-type & RB2575 & RB2575 & RB2575 & RB2575 & RB2575 & RB2575 & RB2575 & RB2575 & RB2575 \\
\hline KHR066 & Wild-type & Wild-type & Wild-type & Wild-type & Wild-type & Un & RB2575 & RB2575 & RB2575 & RB2575 \\
\hline KHR069 & Wild-type & Wild-type & Wild-type & Wild-type & Wild-type & Unknown: & RB2575 & RB2575 & nnọvin & Wild-type \\
\hline
\end{tabular}

F

$6.13 \mathrm{cM}$

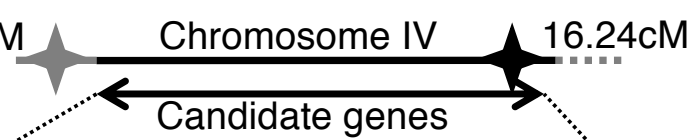

Gene

irg-6

tbc-9

F55B11.1

tag-273

\section{Mutation site}

Splicing site

Amino acid change

Splicing site \& amino acid change

Amino acid change

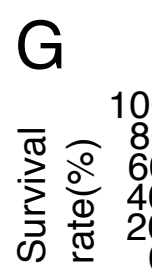

$\mathrm{H}$

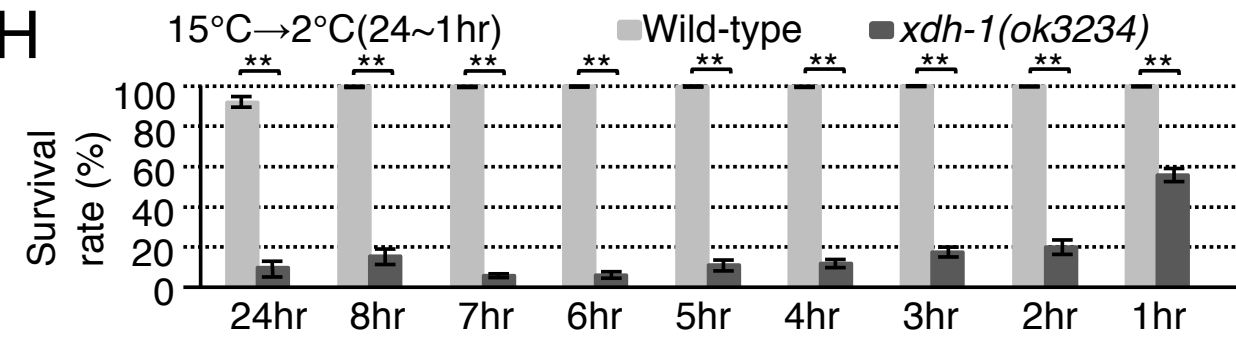




\section{Figure S2}

A

\begin{tabular}{|c|c|}
\hline $\begin{array}{l}\text { Iron-sulfur } \\
\text { cluster }\end{array}$ FAD & Molybdopterin \\
\hline \begin{tabular}{|l|l|} 
& $37 \% / 52 \%$ \\
\end{tabular} & $49 \% / 60 \%$ \\
\hline
\end{tabular}

Conserved sequence $1: 58 \% / 81 \%$

Conserved sequence $2: 74 \% / 82 \%$

\begin{tabular}{|l|l|}
\hline $\begin{array}{l}\text { C. elegans XDH-1 } \\
\text { Vs }\end{array}$ & $\begin{array}{l}\text { Whole sequence: } \\
\text { Identity : } 47 \%\end{array}$ \\
$\begin{array}{l}\text { Homo sapiens XDH } \\
\text { (NP_000370) }\end{array}$ & Similarity : 59\% \\
\cline { 2 - 2 } &
\end{tabular}
B
chr1:
chr1: Splice
D29N
Conserved sequence 1
acceptor site

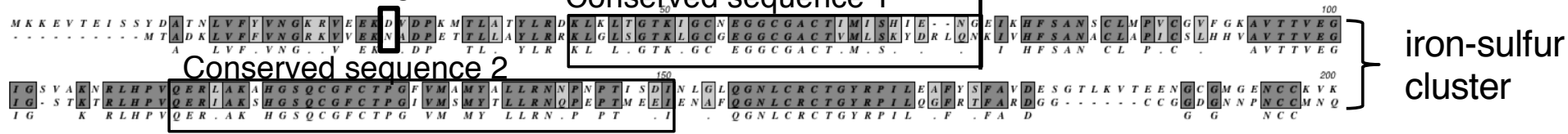

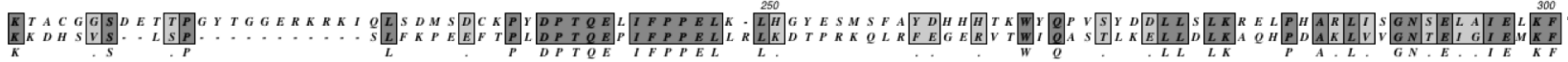

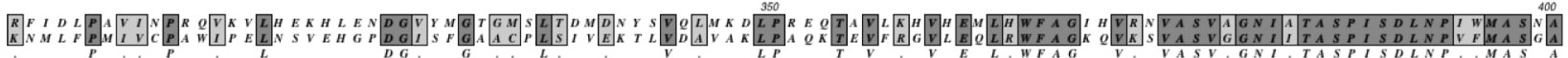

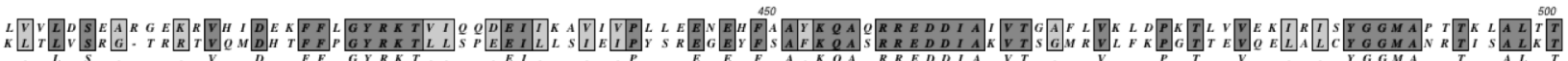

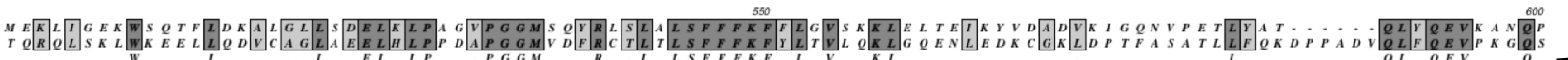

FAD (NAD biding site)

- ok3234: deletion

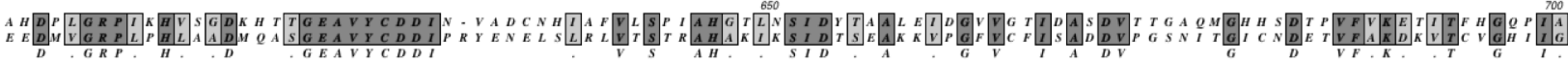

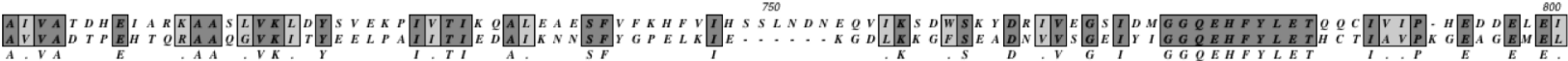

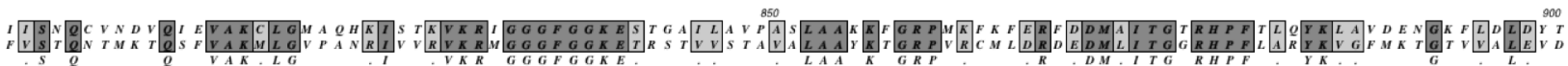

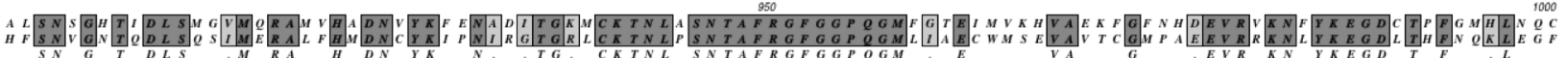

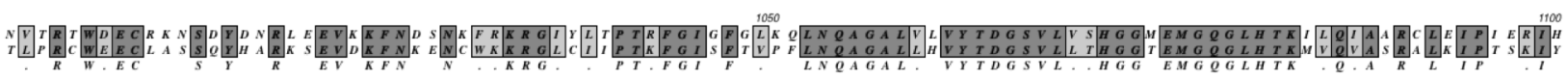
Molybdopterin redox center

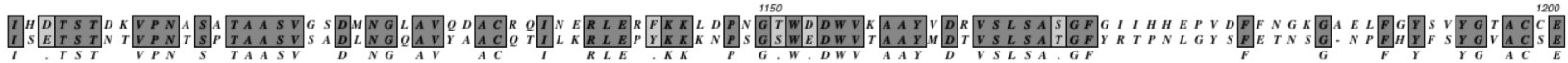

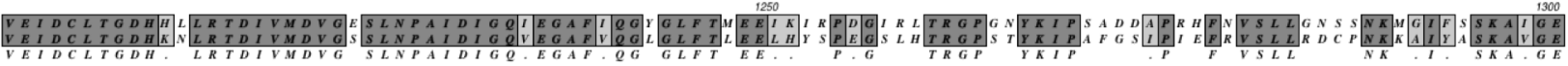

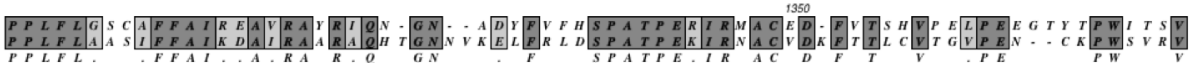
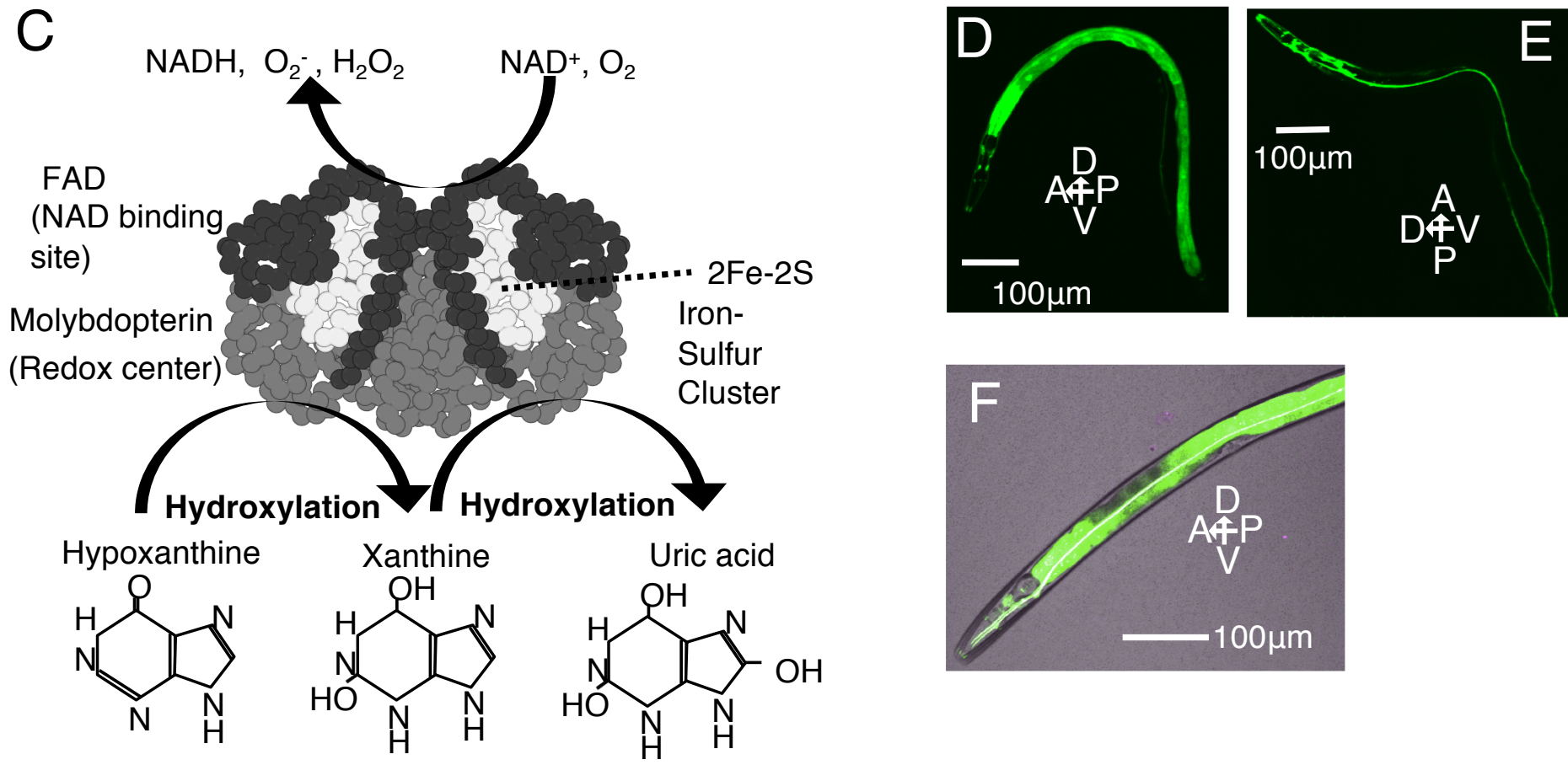

Iron-
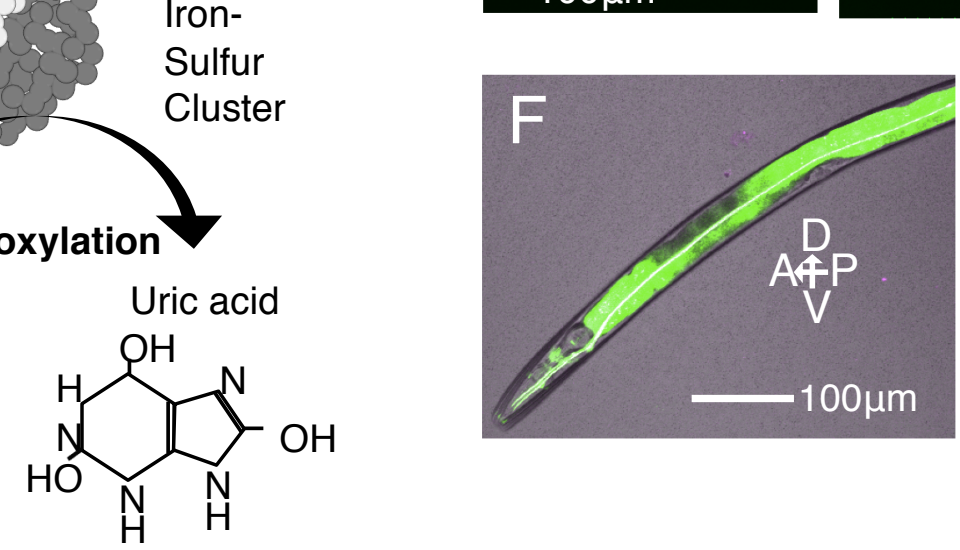


\section{Figure S3}
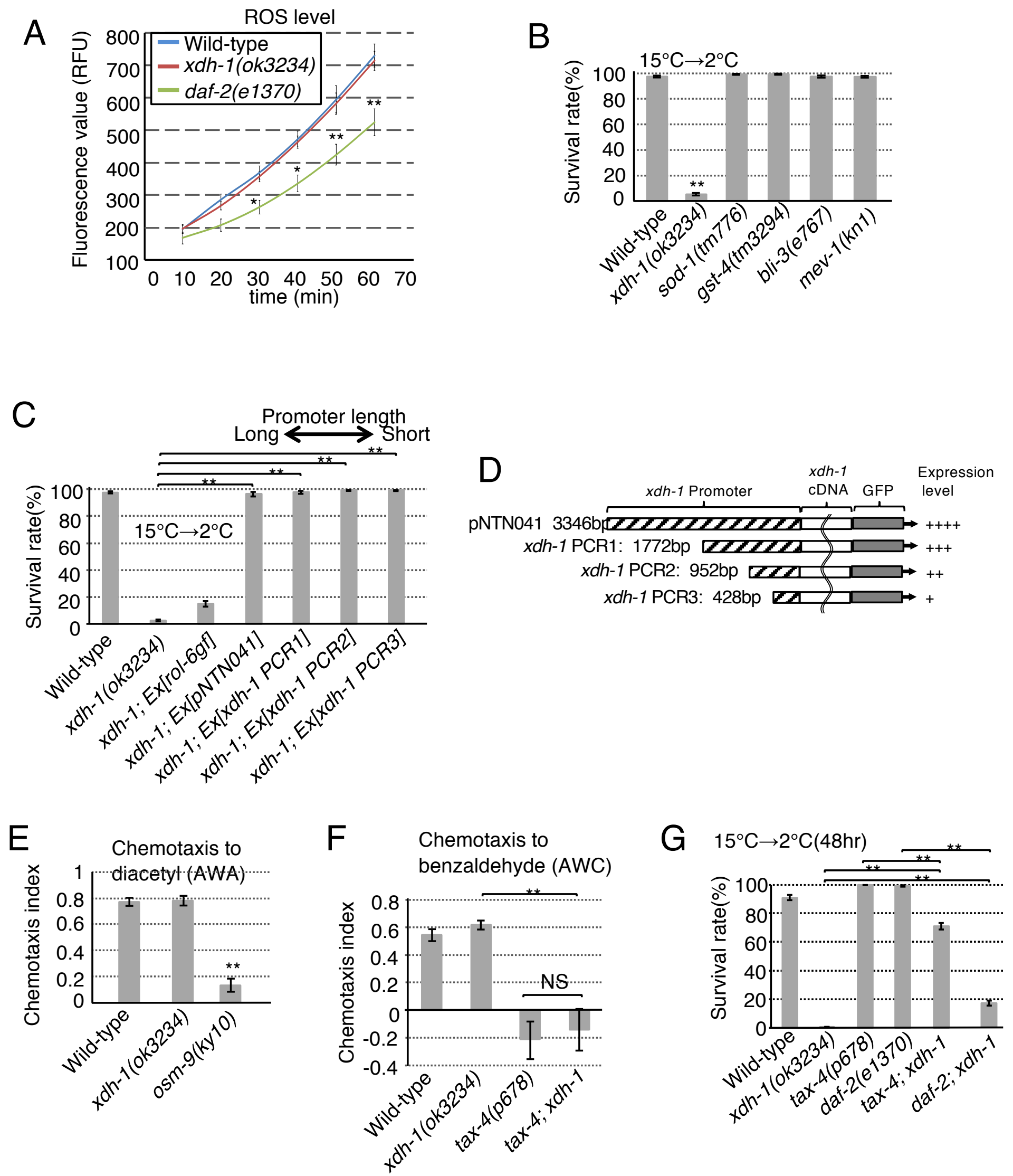


\section{Figure S4}
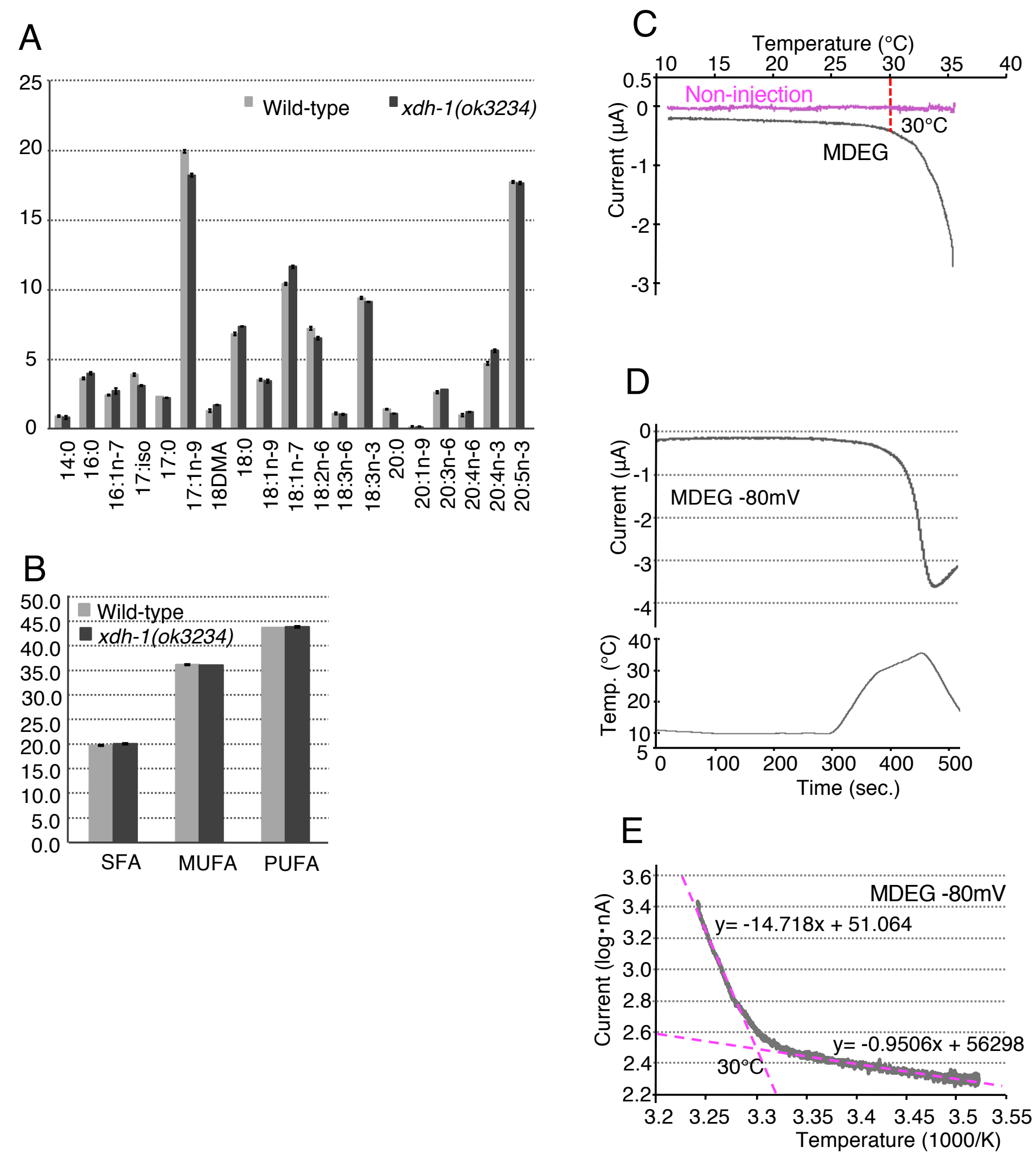